\title{
Tendencias de la radio online como apoyo a la construcción de estrategias propias para el medio*
}

\author{
Jenny Marcela Cardona Bedoya** \\ Yadira Carolina Vaca González ${ }^{* * *}$ \\ Recibido: 2018-09-02. Enviado a pares: 2018-09-15. \\ Aprobado por pares: 2018-11-03. Aceptado: 2018-11-28 \\ https://doi.org/10.22395/angr.v17n34a9
}

\begin{abstract}
Resumen
El desarrollo del proyecto Tendencias de la radio online como apoyo a la construcción de estrategias propias para el medio, presenta a través de la revisión de literatura, el análisis de tendencias que impactan la radio a nivel digital para facilitar la formulación estratégica de las emisoras online a partir de la identificación de tendencias, la transformación del medio y el reconocimiento de acciones digitales. La propuesta metodológica se desarrolló a través de la matriz Micmac ${ }^{1}$ en la que se consideran tres categorías: 1) características de la radio como medio de difusión, 2) estrategias digitales de consumo y 3) la relación de la audiencia con el medio. El planteamiento de las categorías facilita el proceso de recopilación de tendencias e información para su correspondiente aplicación. Se utilizaron fuentes secundarias como IAB, Hubspot, Revista PEM, entre otras, teniendo como referencia la emisora digital 306Radio. Resultado de aplicar la matriz, se encontraron tres tendencias que impactan la radio a nivel digital. En primer lugar, audio programático, en segundo lugar, mezcla de programaciones y, en tercer lugar, el branded content radiofónico. Estas tendencias exploradas en un contexto real, facilitan el planteamiento estratégico mediante el apoyo de las nuevas tecnologías para el medio.

Palabras clave: medios de comunicación; radio; audio; contenidos; tecnología; audiencia; estrategias de comunicación; sonido.
\end{abstract}

Este artículo se origina en el proyecto de investigación Impacto de las tendencias en la transformación de los medios de comunicación de la Facultad de Comunicación de la Fundación Universitaria Unipanamericana, encaminado a analizar las tendencias de la radio online.

** Magíster en Gestión y Dirección de Marketing Global y Nuevos Mercados, especialista en Gerencia en Publicidad, profesional en Mercadeo y Publicidad, experta en la identificación de necesidades y exigencias de empresas vinculadas a diferentes sectores económicos y generación de estrategias para hacerlas competitivas en un mercado global, dirección de proyectos de investigación, Facultad de Comunicación, tutora virtual de la Fundación Universitaria Panamericana, Bogotá, Colombia. jennycardona@unipanamericana.edu.co. Orcid: https://orcid.org/00000002-0453-4304. Google académico: https://scholar.google.com/citations?user=sX66I-UAAAAJEhl=es

.** Magíster en Gestión y Dirección de Marketing Global y Nuevos Mercados; especialista en Gerencia en Publicidad; profesional en Mercadeo y Publicidad de la Fundación Universitaria Panamericana, Bogotá. yadiravaca@ unipanamericana.edu.co. Orcid: http://orcid.org/0000-0001-8606-9903. Google académico: https://scholar. google.es/citations? user=RMai7toAAAAJEhl=es

1 El acrónimo Micmac proviene de las palabras: Matriz de Impactos Cruzados Multiplicación Aplicada a una Clasificación, método elaborado por M. Godet en colaboración con J.C. Duperrin de acuerdo a Godet (2007, p. 65). 


\title{
Trends in online radio as a way of supporting the construction of strategies tailored for the medium
}

\begin{abstract}
By means of a literature review, this paper presents an analysis of trends that impact the radio at digital level to facilitate the strategic formulation of online stations from the identification of trends, the transformation of the medium and the recognition of digital actions. The methodological proposal was developed with the Micmac matrix ${ }^{2}$ in which three categories are considered: 1) characteristics of the radio as a means of diffusion, 2) digital consumption strategies, and 3) the relationship between audience and medium. This approach facilitates the process of gathering trends and information for its implementation. Secondary sources such as IAB, Hubspot, and P \& M Magazine, among others, were used, taking the 306Radio digital station as a reference. As a result of using the aforementioned matrix, three trends that impact the radio at digital level were found. First, programmatic audio; secondly, programming mix; and thirdly, radio branded content. These trends, explored in a real context, facilitate the strategic approach through the support of new technologies for the medium.
\end{abstract}

Keywords: media; radio; Audio; contents; technology; audience; communication strategies; sound.

\section{Tendências da rádio online como apoio à construção de estratégias próprias para o meio}

\begin{abstract}
Resumo
O desenvolvimento do projeto "Tendências da radio online como apoio à construção de estratégias próprias para o meio" apresenta, por meio da revisão de literatura, a análise de tendências que impactam a rádio digitalmente para facilitar a formulação estratégica das emissoras online a partir da identificação de tendências, da transformação do meio e do reconhecimento de ações digitais. A proposta metodológica foi desenvolvida por meio da matriz Micmac ${ }^{3}$ na qual são consideradas três categorias: 1) características da rádio como meio de difusão, 2) estratégias digitais de consumo e 3) a relação da audiência com o meio. A apresentação das categorias facilita o processo de recompilação de tendências e informação para sua correspondente aplicação. Foram utilizadas fontes secundárias, como IAB, Hubspot, Revista PEM, entre outras, tendo como referência a emissora digital 306Radio. Depois de aplicar a matriz, foram encontradas três tendências que impactam a rádio digitalmente. Em primeiro lugar, áudio programático; em segundo lugar, mistura de programações e, em terceiro lugar, o branded content radiofônico. Essas tendências, se exploradas em um contexto real, facilitam a abordagem estratégica por meio do apoio das novas tecnologias para o meio.

Palavras-chave: meios de comunicação; rádio; áudio; conteúdos; tecnologia; audiência; estratégias de comunicação; som.
\end{abstract}

The Micmac acronym comes from "Cross-impact matrix multiplication applied to classification", which is a method developed by M. Godet in collaboration with J. C. Duperrin (Godet, 2007, p. 65).

O acrônimo Micmac provém das palavras: Matriz de Impactos Cruzados Multiplicação Aplicada a uma Classificação, método elaborado por M. Godet em colaboração com J.C. Duperrin de acordo com Godet (2007, p. 65). 


\section{Introducción}

Teniendo en cuenta los cambios tecnológicos y cómo los medios se han visto sujetos a reestructurar su alcance, es fundamental conocer sus ventajas y debilidades al enfrentar su transformación de lo tradicional a lo digital ¿Cuál es la viabilidad a nivel de adaptación frente a las nuevas tendencias? Este crecimiento ha condicionado los hábitos y las costumbres de los usuarios. La conectividad forma parte del día a día de los nuevos consumidores "estamos ante un mundo en cambio, en cambio por la conectividad que se nos ofrece, conectividad entre personas, pero también entre personas y objetos o incluso entre objetos (luminarias)" (Socelec, 2017).Si bien estos representan avances, es necesario saber cómo cada medio puede adaptarse a ellos de la mejor manera y cómo se genera una ventaja competitiva frente a los demás.

La radio ha sido uno de los medios que más ha cambiado y evolucionado ante las nuevas tecnologías, esto ha generado una transición en la que la calidad forma parte principal del cambio respecto al sonido, la estabilidad y la recepción, "si bien el uso de la radio tradicional todavía sigue vigente, el centro de consumo de la radio se trasladó de la casa, un lugar fijo y estable, a las tablets, computadoras o celulares, dispositivos móviles que nos permiten informarnos y entretenernos en tránsito" (Crettaz, 2013). De igual forma, el manejo de estrategias para la vinculación de la audiencia se centra en una comunicación bidireccional que crea un intercambio de información entre emisor y receptor y un diálogo o debate en dicho proceso.

En el caso de la radio online, esta utiliza la tecnología streaming que permite que el audio y el video se complementen con el fin de generar una transmisión en tiempo real. A través de un funcionamiento básico mediante computador se produce una transmisión de audio a un servidor en la web con un software de streaming, este audio no se queda alojado sino que tiene la posibilidd de pasar continuamente para ser escuchado por cualquier persona en tiempo real, se tiene así una cobertura mayor y se llega a cualquier lugar del mundo. Entre sus ventajas se encuentran la disminución por trámites de licencia, cobertura mundial, transmición a bajo costo desde cualquier lugar, esto teniendo en cuenta los requisitos tecnológicos mínimos, la minimización del presupuesto y la generación de estrategias multimedia.

Se busca, entonces, determinar qué tendencias contribuyen a la radio online que permitan la generación de estrategias propias del medio y esto en un acercamiento a la emisora 306Radio. La emisora nace en el 2009 y busca posicionarse como una emisora web para una audiencia juvenil teniendo en cuenta su contenido. Ha trabajado en el desarrollo de contenidos relacionados con su audiencia a través del storytelling o uso de estrategias narrativas, la programación musical y la generación de una comunicación bidireccional, para lograr así la consolidación de su comunidad. La programación incluye como eje las temáticas deportivas, espacios que permiten conocer equipos, jugadores y disciplinas. Se destacan deportes como el fútbol -cuenta con el programa oficial de Millonarios Fútbol Club y del Club Independiente Santa Fé con programas creados desde la hinchada-. De 
igual forma, trabaja contenidos desde Estados Unidos de América, relacionados con el béisbol y el fútbol americano de la NFL. Hay que anotar que el tenis y el golf también forman también parte de su parrilla de programación. En los últimos siete años, se ha consolidado como mejor emisora web en el 2011 por los premios Twitter, en el 2012 premio a mejor contenido deportivo y mejor contenido del año. En el 2013 se generó una diversificación del medio al crear la revista 306 que abarca así nuevos mercados en universidades del territorio nacional. Finalmente en el 2015 nace 306Media Group como una agencia de comunicaciones que busca ampliar el portafolio de servicios audiovisuales de la compañia. Así que se busca indagar ¿Qué tendencias impactan la radio a nivel digital para facilitar la formulación estratégica de las emisoras online? teniendo presente la identificación de tendencias y su incidencia en la radio online, las transformaciones del medio y finalmente el reconocimiento de las estrategias digitales para su aplicación en el sector.

\section{Metodología}

La investigación es de tipo exploratorio, con el fin de poder recopilar información sobre las tendencias que impactan la radio online a partir de la ejecución de la matriz Micmac. Para su aplicación se inició con el planteamiento de tres categorías de análisis que permiten la recopilación de tendencias. Estas categorías se formularon a partir de los objetivos a lograr con la investigación desde lo general a lo específico: de acuerdo con esto, la primera categoría es la identificación de las características de la radio como medio de difusión de contenidos, la segunda categoría son las estrategias digitales de consumo en el medio y la tercera categoría, es la relación audiencia-medio. Luego de la aplicación de la matriz se reconocieron las tendencias con mayor impacto que guiarán la generación de estrategias para la emisora 306Radio teniendo presente su contexto actual. En cuanto al diseño de investigación, se precisa que es de naturaleza transversal con un período de análisis de seis meses. Como lo menciona Arnau (1996) "un diseño puede ser transversal desde la perspectiva de los objetivos y el tiempo en el que se determina" (p.7) lo que permite identificar a nivel conceptual las tendencias manejadas y su incidencia en la emisora analizada.

Al generar un análisis de las tendencias seleccionadas se buscó explorar la radio online desde distintas perspectivas, las cuales se complementan para tener una visión general del medio. Se partió de conocer la radio, su contexto y cómo a partir de la evolución a nivel tecnológico del medio se ha adaptado a las distintas plataformas digitales. De igual forma, se buscó identificar el medio desde lo digital, cuáles han sido las transformaciones, cambios y desarrollos tecnológicos. Finalmente, se analizó la audiencia desde sus características psicográficas y conductuales: gustos, necesidades, hábitos y comportamientos de consumo a nivel digital. Anticiparse a los cambios del mercado es un elemento determinante para las organizaciones con el fin de fortalecer el posicionamiento de las marcas, consolidar la relación con los consumidores e identificar oportunidades para captar nuevos mercados y públicos.

Las distintas categorías de mercado en la que se encuentran los productos y servicios, constantemente envían señales a las organizaciones y a los cazadores de tendencias 
también conocidos como Coolhunters, estos tienen como objetivo reconocer las señales de los consumidores en el mercado y generar acciones de manera paralela. De esta forma, en el día a día, se construyen las tendencias del mercado, las cuales se generan a nivel macro, pero se expresan en los hábitos, comportamientos y conductas, por lo cual las marcas estarán en capacidad de identificar y captar distintas oportunidades que no solo faciliten la toma de decisiones, sino que permitan guiar las acciones a nivel estratégico. En el momento en que las compañías, productos y servicios estén en sintonía con el mercado tendrán mejores posibilidades de crecimiento, visibilidad y posicionamiento ante su competencia.

\section{Discusión: La radio online un medio para crear}

Desde sus inicios la radio ingresó a los hogares de los ciudadanos como un medio de proximidad. Este contaba con la capacidad, en cuanto a la comunicación, de posibilitar la interactividad entendida como conversación, el manejo del lenguaje oral, el apoyo de una comunicación precisa y el desarrollo de una escritura apropiada invitando a la reflexión, generando una relación directa con los usuarios. En un principio, su objetivo se centraba en llevar el mensaje a sus oyentes, sin embargo, con el pasar de los años surgió la necesidad de generar contenidos más precisos según el público objetivo. De esta forma poco a poco se masificó, dando validez a la información brindada:

Con la radio uno está construyendo continuamente imágenes mentales. Defiendo la fantasía como un elemento clave para acercarme a la realidad. Los que hemos mamado de la radio, la música y de los libros tenemos más capacidad de acercarnos a la realidad que los que están hipnotizados por la evidencia visual (García y García, 2001).

Teniendo en cuenta los cambios entre la tecnología análoga y la digital a mediados de la década de los noventa, las emisoras generaron un proceso de cambio y se expandieron a través de nuevas plataformas de difusión que ampliaban su cobertura buscando de esta forma nuevas alternativas en su oferta:

Los cibermedios alteran los modelos comunicativos al incorporar plenamente los procesos de interactividad de los emisores con los usuarios. La interactividad se instala como un proceso multidimensional que va desde los aspectos de las mediaciones tecnológicas hasta la expresión multimedia. Con la interactividad, los cibermedios y los usuarios modifican sus relaciones (Herreros, 2009, p. 17).

Es así como se han generado nuevas técnicas de interactividad, nuevas relaciones entre el programa y sus oyentes, el cambio en la forma de brindar la información y el manejo en los modelos narrativos. La radio se ha caracterizado por su dinamismo, crecimiento y versatilidad. En los últimos años se han adoptado nuevas tecnologías y estas se han aprovechado a favor del medio. Sin embargo, la radio debe estar en constante cambio teniendo en cuenta el público objetivo al que va direccionada, un público que busca formarse, informarse y entretenerse dando una mirada cada vez más crítica frente a lo que se le presenta. De igual forma: 
Las nuevas tecnologías suponen, pues, un cambio fundamental en el concepto, uso y tratamiento de la radio, que se transforma, con la ciberradio, en un nuevo ser, distinto del que era, ya que adquiere imagen y texto, pero no por ello debería perder su identidad de comunicación sonora en aras de una aparente virtualidad de su dimensión como expresividad visual (González, 2010, p. 52).

A su vez, las tendencias a las que se enfrenta la radio online proponen cambios y la generación de alternativas que contribuyan a la competitividad.

Entre las ventajas de la radio online se encuentra: ampliación de la cobertura ya que no existen límites geográficos; especialización en el consumo de los mensajes teniendo en cuenta el contexto; plataformas universales relacionadas con el alojamiento de la información; convergencia en la relación de las terminales y dispositivos utilizados según el consumo; los reenvíos compartidos y modificados y, finalmente, el enfoque multimedial en el consumo de los mensajes, como lo menciona Orihuela:

Los medios cambian cuando cambia la cultura de la audiencia, y aunque el cambio esté inducido por la tecnología, su naturaleza es más profunda: tiene que ver con los nuevos modos en los se produce, se distribuye y se consume la información (2015, p.22).

Es por esto que la radio ha buscado estar a la vanguardia de las nuevas tendencias y generar un vínculo cada vez más estrecho con su audiencia. Martínez y Prata (2017) generaron un comparativo entre los modelos de la radio tradicional versus la radio digital, a continuación, se presenta en la tabla 1 .

Tabla 1. Modelos de comunicación radiofónica

\begin{tabular}{ll}
\hline \multicolumn{1}{c}{ Modelo tradicional de comunicación radiofónica } & Modelo digital y convergente de comunicación radiofónica \\
\hline Público & Comunidad \\
\hline Masivo & Personalizado \\
\hline Pasivo & Activo \\
\hline Receptor & Distribuidor/productor \\
\hline Vertical & Horizontal \\
\hline Linear sincrónico & No-linear/diacrónico \\
\hline Continuidad & Fragmentación \\
\hline Proximidad & Involucramiento \\
\hline Sintético & Expandido \\
\hline Apenas audio & Multimedia \\
\hline Cerrado & Abierto \\
\hline Estandarizado & Experimental \\
\hline
\end{tabular}

Fuente: Martínez y Prata, 2017. 
Es de esta manera que la radio genera unos cambios significativos, pasando de comunicar a un público a generar comunidades, de ser masivo a trabajar con nichos específicos del mercado y de esta forma el oyente es un actor cada vez más participativo, se cambia la comunicación vertical por horizontal, se vincula al público a través de programaciones multimediales en los que la experiencia forma parte importante del proceso.

\section{La transformación del medio a nivel tecnológico y cultural}

La radio ha superado múltiples desafíos, en los que se destacan desafíos tecnológicos y culturales. El primero de ellos alude a cómo el impacto social ha generado cambios significativos en el medio online, favoreciendo la generación y producción de contenidos de manera integral entre la oferta realizada y su audiencia, involucrándolos para la generación de experiencias únicas, diferentes e innovadoras, entendiendo la realidad y sus necesidades. Sin embargo, no se puede olvidar que el tema de conectividad no impacta a todos los sectores o poblaciones en una ciudad o país, dado que se deben tener una serie de requisitos y condiciones mínimas para su acceso, de manera que es "un aspecto clave en las estrategias de difusión y recepción de nuevos contenidos radiofónicos" (Romero, 2011) generar el análisis de la población con el fin de conocer a fondo qué se requiere y cómo se pueden lograr los objetivos determinados por el medio.

Los cambios generados por las Tecnologías de la Información y de la Comunicación (TIC) han forjado un modelo donde la radio es más activa, abierta, personalizada y experimental, donde la red ha cambiado la distribución tradicional por una bidireccional, en donde los oyentes pueden navegar en un espacio multimedia y elementos visuales y sonoros se unen para generar una exploración más amigable y amena, vinculando de manera directa al público objetivo y haciendo que este forma parte activa de la comunidad:

Puede decirse que la naturaleza multimedia e interactiva de la red amplía el espacio comunicativo entre una cadena de radio y su público. Además, internet se presenta como una ocasión para enriquecer las estrategias discursivas del medio radiofónico, desarrollar contenidos más personalizados e intensificar el diálogo con el grupo de oyentes que se identifican con un estilo particular de hacer radio (Costa, Moreno y Amoedo, 2012).

Así, se puede afirmar que internet es la herramienta pero el cómo se use forma parte del equipo de trabajadores y los objetivos propuestos por la organización.

El desarrollo de programas que sean pensados para interacturar de manera cons tante con el oyente a través de las herramientas propuestas; la generación de elementos creativos que diferencien la programación y su vinculación con el oyente sumados a los paradigmas de la radio online-que poco a poco fue rompiendo barreras preconcebidas como el cambio de un sistema de alambres a uno por fibra óptica-; la modificación del concepto de territorialidad a un concepto global; la programación trabajada desde nichos especializados; todo esto ha hecho que la tecnología forme parte importante del medio y de sus avances: 
El consumidor tradicional de medios ahora es un sujeto activo que, además de desarrollar competencias interpretativas cada vez más sofis-ticadas para comprender los nuevos formatos narrativos, de manera cre-ciente crea nuevos contenidos, los recombina y comparte en las redes digitales (Scolari, Winocur, Pereira, y Barrechene, 2018).

Si bien existen grandes fortalezas en el manejo online, no se pueden dejar de lado las barreras del uso tecnológico en el medio, la conectividad, el idioma, el manejo multimedia o la fidelización a la audiencia son algunas a los que el medio se puede ver enfrentado. En el caso de la conectividad, se puede mencionar que no todos tienen acceso al medio y algunos lo tienen con restricciones, por ejemplo en el caso de Colombia:

Al cierre del tercer trimestre del año 2017, el número total de accesos a internet en el país alcanzó los 16.635.789, cifra compuesta por accesos a internet fijo y móvil, lo que representó un índice de penetración nacional de accesos a internet del 33,7 \%, y un aumento de 3,6 puntos porcentuales con relación al índice del tercer trimestre del 2016 (MinTIC, 2018).

Si bien se ha crecido en este aspecto, aún falta eliminar las brechas de accesibilidad. En el caso del idioma, las personas buscan contenidos preferiblemente en su mismo idioma lo que en ocasiones limita la cobertura. En cuanto al manejo multimedia es necesario que se tenga un equipo de profesionales que conozcan el medio y cómo debe generarse la difusión, de esta forma se puede generar una vinculación para la relación de fidelización con el usuario y que este no solo este de "paso" en la emisora o en los programas de la misma sino que pertenezca a ella, genere contenido y se vincule con su crecimiento. En cuanto a los procesos culturales en los que los valores individuales y colectivos entran en acción para establecer patrones de comportamiento que generan el desarrollo social de los individuos, es necesario entender estas diferencias culturales, lo que permite el enriquecimiento de las temáticas y contenidos a desarrollar en la radio online, es claro que la manera de escuchar se ha transformado desde las plataformas, tiempos y momentos, al punto que nunca serán los mismos de manera que cada vez más se está expuesto a una necesidad social que pretende la gestión estratégica de los procesos culturales, hoy en día conocido como las industrias culturales y creativas presentada por la Unesco.

Levy (2007) menciona que:

Los nuevos usuarios, habitantes de la nueva cultura digital, están cambiando la forma tradicional de abordarla. Hasta hace poco, sólo los artistas, escritores, académicos,... participaban en la creación de referentes culturales, en el contenido y en las formas de manifestar saberes individuales y grupales; hoy no, cada vez más los elementos de la cultura y los conocimientos sobre ellos son proporcionados por una ciudadanía participativa ajena a las mencionadas élites de decisión (p.296).

Las TIC apoyan esa relación empresa-audiencia, de manera tal que la comunicación sea eficaz siendo consecuentes con las necesidades. 
La radio cumple un papel trascendental como un medio dinamizador de lo cultural y lo social, esto teniendo en cuenta que la mayoría de los oyentes se informan y entretienen. La cercanía y empatía con el medio se da cuando el oyente genera interacción, cuestiona, participa y debate, sin embargo, si se tiene en cuenta la cobertura presente, es necesario que los empresarios generen una investigación de sus públicos, estén atentos del seguimiento a los mismos y a las participaciones realizadas de tal forma que los objetivos y estrategias estén direccionados a las metas propuestas. La sociedad actual está inmersa en constantes cambios culturales, si bien aún lo análogo juega un papel importante en la vida diaria de la audiencia, el internet se ha convertido en una herramienta que toma más fuerza imponiéndose ante las nuevas tendencias, lo que plantea, de esta forma, nuevos retos para las empresas y sus oyentes, "las TIC en general permiten a los usuarios la posibilidad de participación al abrir espacios en los cuales pueden proporcionar información, complementar datos expuestos por otros, comentar la postura de uno u otro e incluso votar por lo allí expuesto" (Barrios, 2013)

\section{El apoyo de estrategias y tendencias en la implementación de la radio online}

La radio como medio poco a poco se ha transformado, lo que inquieta a los empresarios según los proyectos futuros, y de cierta forma genera una reflexión: ¿Estamos preparados para estos cambios? ¿Qué cambios significativos se han generado y cómo podemos estar a la vanguardia de ellos? ¿Qué significa este cambio en el medio y en los demás medios? Un sin número de preguntas nos podemos hacer, y una de ellas está relacionada con las estrategias que como organización debemos implementar teniendo presente las nuevas tendencias, es por esto que nos vemos obligados a revisar cómo nos enfrentamos a dichos cambios y si las estrategias que se han generado no solo están encaminadas a nuestros objetivos, sino si están vinculadas directamente con la audiencia.

Teniendo en cuenta las características del medio podemos tener presente en cuanto a la estrategia: primero tener una óptima calidad en la transmisión y en la música emitida, este es un elemento importante teniendo en cuenta procesador, servidor, memoria y ancho de banda lo que permitirá una transmisión de calidad. En segundo lugar, se encuentra el uso de redes sociales, pero no es solo crear las cuentas sino saberlas manejar, es necesario elegir las más apropiadas según el público objetivo al que se va dirigido, esto apoya un marketing digital estratégico y permite una relación estrecha con la audiencia, convirtiéndola en parte de la comunicación. En tercer lugar, tener presente a la emisora en directores especializados lo que apoyará su consolidación, ampliando su cobertura, esto teniendo en cuenta la ventaja competitiva con la que cuente la marca. En cuarto lugar, se encuentra potencializar la audición teniendo presente una diversidad en la programación, los invitados, las temáticas abordadas y la manera en que nos comunicamos con la audiencia. En quinto lugar encontramos la interactividad y el dinamismo, este es un elemento fundamental si se quiere que la audiencia participe en las actividades propuestas y genere un vínculo directo con la emisora. Por último, en sexto lugar se encuentra la 
permanencia que se afecta por los continuos cambios generados, por lo tanto se debe tener presente qué se quiere y cómo se quiere lograr.

Estas estrategias deben estar vinculadas directamente con las tendencias del medio, por ejemplo la Corporación Colombia Digital (2017) menciona cinco tendencias que se deben tener presentes en la radio online:

Transmisión IP: si bien se genera una cobertura cada vez más amplia es necesario tener presente los nichos específicos a los cuales va direccionada la emisora de acuerdo con la relación con la audiencia.

- Una radio visual: el aprovechamiento del video en la radio permite una relación más cercana con sus oyentes, lo que genera un aprovechamiento en el relacionamiento con otros medios, sin ser este su fuente principal.

El podcast: el apoyo en la distribución de archivos multimedia con piezas visuales de corta duración que incluyen subtítulos, textos y notas, esto apoya el fortalecimiento radiofónico.

- La radio digital: apoya la modernización de la señal y genera una mayor calidad en la optimización de la emisora.

El apoyo transmedia: la incorporación de otros medios y la vinculación de los mismos en la creación de historias permite un vínculo más directo con la audiencia y la participación de la misma.

\section{Perspectiva metodológica del proyecto}

La articulación entre el mercado, el consumidor y los medios es el diferencial que pueden desarrollar las emisoras online, reflejado en la construcción y diseño de la parrilla de programación y cada uno de los programas a emitir, el entender las necesidades de la audiencia y la capacidad del medio son factores a tener presentes en el planteamiento de las acciones que permitirá fortalecer el posicionamiento digital de las emisoras. Es por esto que el propósito de la investigación se centra en analizar las tendencias que impactan la radio en lo digital para facilitar la formulación estratégica de las emisoras online. Para lograrlo se inició con la identificación de tendencias digitales en el medio con el fin de determinar su incidencia en la radio online, después de esto se buscó distinguir las transformaciones del medio desde lo tecnológico y digital para, finalmente, reconocer las estrategias digitales a utilizar con el fin de orientar las acciones del medio online.

En relación con el proceso metodológico se establecieron las tendencias que impactan la radio online y, paralelamente, la evolución de las estrategias de marketing digital. A través de la aplicación de la matriz Micmac se generó un análisis estructural de escenarios y estudios futuros, lo que permite la toma de decisiones en relación con las necesidades existentes. Una vez son obtenidos los resultados después de la identificación de las tendencias, se generan estrategias de impacto para el proyecto teniendo como caso de estudio la emisora 306Radio. 
En relación con el proceso de recopilación de tendencias, se realizó una exploración de fuentes secundarias desde tres categorías de análisis, la primera identifica el medio de comunicación desde la perspectiva de la radio online como base del proyecto, el segundo las estrategias de marketing digital teniendo en cuenta su impacto en el medio y el tercero la audiencia y su relación directa con la radio online. Realizada la exploración se dio inicio al análisis desde la matriz Micmac la cual se desarrolla en cinco fases, la primera reconoce el inventario de las tendencias en relación con la radio online y su impacto a nivel actual desde la perspectiva nacional como internacional, la segunda relacionada con la descripción de dichas tendencias de acuerdo con el grado de relación con el tema de análisis, en tercer lugar se seleccionan las tendencias de mayor viabilidad, la cuarta fase es la matriz de impactos cruzados donde se puede generar una evaluación de 0 a 3 teniendo en cuenta la dependencia e influencia de las mismas, por último, en la quinta fase se genera clasificación de resultados y se llevan a una gráfica que permite reconocer las tendencias ganadoras.

\section{Hallazgos: proyecto realizado a través de la matriz Micmac -306Radio}

\section{Planteamiento de las categorías de información para la búsqueda de tendencias}

Actualmente, a través de cada medio de comunicación se emiten mensajes en diferentes horarios, momentos y plataformas, que no solo pretenden impactar sino persuadir al consumidor a reconocer y adquirir las distintas marcas. Estos cambios son el resultado de transformaciones generadas por el mercado, las marcas, los medios y la audiencia, por ello el identificar y analizar las tendencias permite descifrar el impacto en la generación de nuevos comportamientos, consumo de marcas, identificación de oportunidades, generación de nichos, por lo tanto, a través de la investigación se busca diagnosticar la categoría de mercado de la radio online para la formulación de acciones estratégicas.

El proceso de recopilación de tendencias parte del planteamiento de categorías de análisis a partir de los objetivos de investigación para delimitar desde el inicio de la matriz las tendencias que puedan tener un impacto a la investigación, se inicia de lo general a lo específico abarcando distintas perspectivas de la radio online. Las tres categorías de análisis que se plantean, se inician con la identificación y análisis de las tendencias que están impactando la radio, seguido de las tendencias en relación con el consumo de medios online y finalmente las tendencias vinculadas al oyente online, es importante resaltar que las tendencias que se describen a continuación se recopilan de fuentes secundarias como IAB reconocida dentro del mundo digital como una plataforma con contenido digital sobre el marketing y publicidad online, Hubspot como plataforma de Inbound Marketing que facilita a las empresas incrementar las visitas, generar leads y lograr nuevos clientes y la Revista PEM es un medio editorial que se especializa en la publicidad y el mercado, a partir de artículos, reportes e investigaciones en relación con la radio online, estas son las 30 tendencias seleccionadas y su correspondiente análisis. 


\section{Primera categoría de análisis: características de la radio como medio de difusión de contenidos}

Esta categoría presenta las tendencias que forman parte de las transformaciones a las cuales está expuesto el medio y los cambios que ha generado para adaptarse a las cambiantes necesidades de los oyentes desde la adaptabilidad del medio como en la generación y difusión de múltiples contenidos, gran parte de los cambios se deben particularmente a aspectos económicos y digitales los cuales han marcado el desarrollo, crecimiento y características de este. Las tendencias que se identifican en la categoría son:

1. Pluralismo de los medios de comunicación: el acceso a los distintos contenidos, plataformas, medios y canales se ha incrementado en los últimos años permitiendo que gran parte de la población mundial tenga acceso. El desarrollo de contenido informativo crece debido a la interacción de los usuarios para compartir, comentar y publicar la información.

2. Independencia de los medios de comunicación: resalta el impacto de los procesos políticos, regulatorios y comercial para los medios de comunicación, en donde se busca un nivel de independencia en su funcionamiento y administración de contenidos pero que a su vez logren la confianza de los públicos, por ello cada vez más los medios propenden por ofrecer un valor añadido soportado en procesos de investigación para la generación de información y contenidos de valor.

3. La radio visual: ofrece una nueva mirada de la radio al presentar a nivel visual el día a día de lo que sucede en la cabina, la transmisión de los distintos programas, lo que le da al medio una nueva mirada ante los oyentes y tiene "el reto de solidificarse como un medio que puede valerse de otros para transformarse" (Bitar, 2018).

4. La radio en el mundo transmedia: presenta la oportunidad de generar nuevas narrativas y ser parte de una campaña de comunicación global fortaleciendo las acciones desarrolladas en los otros medios de comunicación. La radio se presenta como el aliado perfecto para crear mundos sonoros, musicales que seduzcan al público de una manera diferente a lo que se está acostumbrado.

5. Mejora de la calidad de la transmisión: permite mejorar la calidad del audio al digitalizar la señal, con el fin de ofrecer productos y contenidos de calidad a los múltiples oyentes.

6. Amplificadores de sonido: la adaptación de amplificadores habilitados mediante la voz permite la generación de nuevas experiencias en torno a la música, canciones y artistas, lo que significa una oportunidad para la generación de contenidos personalizado con el fin de captar la atención de los nuevos usuarios, reorganizando los momentos y espacios de consumo y generadores de entretenimiento mezclando elementos como luces o calefacción.

7. Múltiples pantallas vinculadas por el sonido: facilita la interacción entre dispositivos para la generación de contenidos audiovisuales, en donde los comandos por voz son más 
rápidos que la escritura en los diferentes dispositivos, los cuales ofrecen una nueva oportunidad para generar videos llamadas, monitoreo, notificaciones.

8. Creatividad en la generación de contenidos: se trabaja en la creación de contenidos que sean innovadores y vayan más allá del uso de los diferentes dispositivos o plataformas, los consumidores pueden estar más involucrados y participar de manera activa con los contenidos y las marcas.

9. Comportamientos cambiantes e intermitentes: los dispositivos están cambiando el consumo de medios del consumidor y los oyentes a nivel online, donde los contenidos multimedia serán los más utilizados para transmitir programas musicales, noticiosos, libros de audio, los cuales permiten incrementar los ingresos de las diferentes industrias.

10. Contenidos en vivo para los medios digitales: presenta cómo los medios se pueden potencializar en un futuro mediante la transmisión en vivo, la radio se caracterizará aún más que la programación bajo demanda, tendrán relevancia los playlist musicales, los cuales dependerán de la conectividad disponible en los espacios en los que se encuentre el oyente.

\section{Segunda categoría de análisis: las estrategias digitales de consumo en el medio}

Estas tendencias se centran en las estrategias digitales que buscan incrementar el consumo del medio online, dentro de estas se distingue un crecimiento constante del uso digital gracias a los múltiples dispositivos móviles que utilizan cada vez más los usuarios y oyentes como plataformas para la exploración y el consumo de diversos contenidos como vídeos, podcast, e-books entre otros, debido a los distintos canales de acceso que cada vez más hacen parte del día a día. Las tendencias que se identifican en la categoría son:

1. Smart audio o audio inteligente: refleja que, a través del uso de múltiples dispositivos, se puede utilizar la voz para el control y generación de acciones de reproducción de sonidos.

2. Podcasting: es una herramienta que permite lograr una conexión más directa con los oyentes mediante la distribución de archivos multimedia, audios digitales sin interrupción de pauta publicitaria o anuncios, lo cuales pueden acceder en cualquier momento de día, en cualquier ocasión y hora.

3. Geolocalización: al ser una estrategia de marketing puede ser muy utilizada y adaptable a los medios digitales y particularmente en la radio online. La geolocalización es cada vez más utilizada en las campañas por ser un medio complementario, en ocasiones puede ser un medio principal para difundir un mensaje logrando nuevos puntos de contacto con el medio o touchpoints, que ofrecen la capacidad de segmentar a través de la "geolocalización, edad, sexo, dispositivo, sistema operativo, horario y género musical, también es posible definir la frecuencia de impacto por individuo" (Tigre, 2018) 
4. Audio 3D: se describe como la optimización del medio para ofrecer más que programas de calidad, en experiencias de escucha innovadoras, en el que se utiliza el rich media o contenidos avanzados con soporte multimedial que no solo atraen al consumidor sino que, además, permiten que interactúen con él, su utilización se facilita en dispositivos y plataformas móviles, al punto que los headphones generation emiten mensajes directos a los oídos de los oyentes.

5. Audio programático: permite comprar audios con publicidad o también conocidos como los audios ads, en los diferentes servicios de streaming como: Google Play Music, Spotify, SoundCloud, entre otros ampliando la oportunidad de monetizar a través de estas plataformas y personalizar los contenidos de acuerdo con las características de la audiencia.

6. Interacciones a través del contenido: no solo se identifican los contenidos que pueden ser relevantes y puedan ofrecer la información deseada por el usuario para lograr persuadirlos e interactuar con la marca a nivel digital.

7. Metatipos sociales: describe cómo a través de los comportamientos del consumidor se están desarrollando y generando distintas adaptaciones del consumo online lo que influye en el desarrollo de la personalidad del individuo, lo que genera la oportunidad de contradecir y eliminar los estereotipos mientras que los metatipos son cada vez más cambiantes y no fieles a una marca.

8. Utilización de influenciadores: los influenciadores son individuos activos para las marcas que presentan un nuevo camino para llegar a los consumidores y reforzar los vínculos, esto depende en gran medida de las características, valores y personalidad del influenciador y cómo puede relacionarse con las marcas. La credibilidad es el mayor atributo que tienen por ello los usuarios los siguen porque hay un gran interés por conocer qué pueden opinar, pensar, percibir sobre un producto, servicio, lugar, entre otros; los influenciados están utilizando cada vez más están utilizando los medios digitales para lograr ese impacto y llamar la atención de más usuarios.

9. Diversificación de los programas radiales digitales: se proyecta un gran crecimiento de las estaciones de radio digitales por la diversidad de contenidos y a los diferentes perfiles que impactan, lo que implica que el medio está al tanto de las necesidades y cambios de los oyentes y a partir de ellas plantear programas y contenido especializados que permitan lograr una conexión duradera con los oyentes.

10. Impacto al crecimiento musical: el desarrollo del medio a nivel digital tiene gran incidencia en la creación de nuevos estilos y artistas musicales, la interacción constante con los oyentes permite una retroalimentación en los ajustes y mejoras a los programas, plataformas y aplicaciones; de manera paralela incentiva y mueve el mercado de los playlist, top 10, lanzamiento de artistas, mezclas y colaboraciones, de manera que el crecimiento de la radio digital tiene in gran impacto y potencial para la generación de nuevas propuestas musicales que sorprendan cada vez más a los oyentes. 


\section{Categoría de análisis: la relación de la audiencia con el medio}

Las tendencias con relación al oyente online permiten lograr un contraste del consumo del medio y los contenidos que se consumen y cómo estos resaltan características de preferencias, gustos, necesidades y formatos. Las tendencias que se identifican en la categoría son:

1. Escuchar audio online: se hace cada vez más frecuente que la audiencia consuma el medio a través de plataformas digitales mediante dispositivos móviles, con la particularidad de la personalización de los contenidos a través de emisoras de radio, servicios de música, listados, top 10 o top 100, en directo por streaming o en descarga. Lo que significa que el medio no está dejando de escucharse, por el contrario, está migrando su consumo a nivel online personalizando completamente su uso y transformarlo bajo demanda o conocidos como on demand.

2. Consumo musical: dentro de las categorías y programaciones más solicitadas y predilectas por los usuarios digitales están los programas musicales, cada vez más ligados con las redes sociales. Permiten interactuar directamente a cada oyente, transformándolo en un prosumidor (productor y consumidor) de contenidos, esto se genera por la facilidad de acceso entre las aplicaciones y los dispositivos que en que se escuchan.

3. Duplicidad auditiva: los oyentes se distinguen por ser multitasking y realizar varias actividades al mismo tiempo, al hablar de los oyentes digitales se identifica una duplicidad con relación a los formatos: la radio directa u online, los listados de música, las emisoras online, en directo, la radio online en diferido, los podcasts/descarga.

4. Personalización del consumo de contenidos: las características psicográficas y conductuales del consumidor influyen en gran medida en la generación de contenidos; los gustos, necesidades y los momentos de consumo son claves y logran el posicionamiento actual del medio. Las razones más determinantes del consumo son: "porque la escucho como y donde quiero, me permite realizar otras actividades, me ofrece una gran cantidad de contenidos" (Acebes y Borrego, 2018)

5. Crecimiento del pago por suscripción: describe uno de los motivos más importante por el cual los oyentes digitales consumirían contenidos y programas online y es el consumo constante ininterrumpido, sin anuncios o interrupciones publicitarias, igualmente ofrece primicias musicales, reconocimiento de nuevos géneros y artistas y escuchar playlist off-line sin necesitar el consumo de datos o sin cobertura.

6. Microsegmentación del target: el consumo de radio online permitirá profundizar en las características de los oyentes a nivel psicográfico y conductual para determinar cuáles son más afines y que perfiles no se están abarcando. Dentro de las características de mayor interés con relación al oyente online se distinguen el género, la formación académica, el núcleo familiar, las motivaciones, los grupos de referencia, entre otros. En conclusión, los oyentes digitales están cada vez más actualizados, tienen acceso 
a mayor formación y un excelente uso de las nuevas tecnologías lo que les permite tener una alta capacidad de adaptación y aprendizaje dentro del medio.

7. Audio en movilidad: describe la alta probabilidad de cobertura que tendrá la radio digital, incrementando el impacto a los oyentes online, describe cómo los contenidos y programaciones digitales se consumirán en distintos momentos, el usuario estará activo y conectado a través de distintas plataformas para no perderse los contenidos de su interés.

8. Mezcla de programaciones: presenta cómo el oyente al personalizar el consumo de contenidos está abriendo nuevas oportunidades relacionadas con el crecimiento del medio, cada vez más será común identificar mezclas de programaciones en vivo y pregrabadas que se adaptan a las necesidades del oyente y establecen una nueva relación para lograr una mayor interactividad y posicionamiento de los programas, el medio y las marcas a nivel online.

9. Storytelling a nivel radial: se trabaja en cómo se pueden contar historias desde las temáticas a trabajar en la programación de las emisoras en línea con el fin de lograr desarrollar escenarios por medio del relato para conectar a nivel emocional por medio de las historias y generar experiencias innovadoras.

10. Branded content radiofónico: la generación de contenidos de marca estarán ingresando a la radio online de manera complementaria con formatos de vídeo con contenidos creativos construidos de manera paralela con las marcas, los cuales ofrecen una nueva oportunidad de pauta y programación a partir en los múltiples segmentos.

\section{Descripción de la relación entre tendencias}

En este segundo apartado de la matriz se realiza el primer filtro de selección a partir de las 30 tendencias anteriormente descritas, mediante el criterio de selección de pertinencia con el objetivo y propósito de la investigación, en promedio se descartan diez tendencias.

\section{Identificación de tendencias con mayor impacto}

En este tercer apartado se realiza el segundo filtro de selección a partir de las tendencias restantes de la fase anterior, los criterios de evaluación para tener en cuenta son la viabilidad de la ejecución de las tendencias y su facilidad en el planteamiento estratégico para lograr transformar una tendencia en una moda. En promedio quedan de siete a diez tendencias. A continuación, se exponen las diez tendencias de las cuales será posible formular acciones estratégicas para la radio online, vinculadas a las distintas categorías de análisis descritas anteriormente (tabla 2). - Tendencias con mayor viabilidad de desarrollo: 
Tabla 2. Tendencias con mayor viabilidad de desarrollo

\begin{tabular}{ll}
\hline \multicolumn{1}{c}{ Tendencia } & \multicolumn{1}{c}{ Categoría de la Tendencia } \\
\hline Audio programático & Tendencias del consumo de medios online \\
\hline El crecimiento del pago por suscripción & Tendencias de oyente online \\
\hline La mezcla de programaciones & Tendencias de oyente online \\
\hline El storytelling & Tendencias de oyente online \\
\hline El branded content radiofónico & Tendencias de oyente online \\
\hline Impacto al crecimiento musical & Tendencias del consumo de medios online \\
\hline Amplificadores de sonido & Tendencias en la radio \\
\hline Audio 3D & Tendencias del consumo de medios online \\
\hline La microsegmentación del target & Tendencias de oyente online \\
\hline La radio visual & Tendencias en la radio \\
\hline
\end{tabular}

Fuente: elaboración propia

\section{Matriz de impactos cruzados}

En la tabla 3 se ven las diez tendencias. En esta cuarta fase de la matriz de manera in dividual se inicia por contrastar la influencia y dependencia que tienen cada tendencia frente a las demás, este proceso se realiza de manera conjunta entre un experto del medio y el investigador al evaluarse cuantitativamente cada tendencia por medio de los valores numéricos 3, 2, 1 y 0 siendo 3 el de mayor impacto, es decir, mayor influencia o mayor dependencia y siendo 0 el de menor impacto, es decir, menor influencia o menor dependencia. A continuación, se listan las 10 tendencias a valorar:

- Tendencia 1. Audio programático.

- Tendencia 2. Crecimiento del pago por suscripción.

- Tendencia 3. Mezcla de programaciones.

- Tendencia 4. Storytelling a nivel radial.

- Tendencia 5. Branded content radiofónico.

- Tendencia 6. Impacto al crecimiento musical.

- Tendencia 7. Amplificadores de sonido.

- Tendencia 8. Audio 3D.

- Tendencia 9. Microsegmentación del target.

- Tendencia 10. Radio visual. 
Para determinar la influencia se inicia de manera vertical de superior a inferior. Por ejemplo, cuál es el nivel de influencia de la tendencia 1 (audio programático) con la tendencia 2 (crecimiento del pago por suscripción) y así sucesivamente hasta finalizar de identificar la influencia de la tendencia 1 con el resto de las tendencias. Se repite el proceso con cada una de las tendencias. Para determinar la dependencia se inicia de manera horizontal de izquierda a derecha. Por ejemplo, cuál es el nivel de dependencia de la tendencia 1 (audio programático) con la tendencia 2 (crecimiento del pago por suscripción) y así sucesivamente hasta finalizar de identificar la influencia de la tendencia 1 con el resto de las tendencias. Se repite el proceso con cada una de las tendencias.

Tabla 3. Matriz de impactos cruzados (MIC)

\begin{tabular}{cccccccccccc} 
& T.1. & T.2. & T.3. & T.4. & T.5. & T.6. & T.7. & T.8. & T.9 & T.10 & D \\
\hline T.1. & & 2 & 1 & 0 & 1 & 0 & 0 & 1 & 1 & 2 & 8 \\
\hline T.2. & 3 & & 1 & 1 & 2 & 1 & 0 & 1 & 1 & 1 & 11 \\
\hline T.3. & 0 & 2 & & 1 & 0 & 1 & 0 & 2 & 1 & 2 & 9 \\
\hline T.4. & 2 & 2 & 2 & & 3 & 1 & 2 & 3 & 2 & 2 & 19 \\
\hline T.5. & 0 & 0 & 1 & 2 & & 1 & 0 & 2 & 1 & 2 & 9 \\
\hline T.6. & 0 & 3 & 3 & 2 & 2 & & 2 & 2 & 3 & 2 & 19 \\
\hline T.7. & 0 & 1 & 0 & 1 & 1 & 2 & & 0 & 1 & 0 & 6 \\
\hline T.8. & 2 & 2 & 2 & 3 & 3 & 2 & 2 & & 0 & 1 & 17 \\
\hline T.9. & 3 & 2 & 2 & 3 & 2 & 3 & 1 & 2 & & 2 & 20 \\
\hline T.10 & 2 & 2 & 2 & 2 & 3 & 2 & 0 & 3 & 2 & & 18 \\
\hline I & 12 & 16 & 14 & 15 & 17 & 13 & 7 & 16 & 12 & 14 & \\
\hline
\end{tabular}

Fuente: elaboración propia

\section{Clasificación de resultados}

Para la tabla de resultados en el plano cartesiano, se selecciona el número mayor del total de influencia o dependencia para tener el número máximo del eje y (influencia) y el x (dependencia), en este caso el número 20. Para la graficación de tendencias se suma el total de la valoración a nivel horizontal y vertical. En la figura 1 se identifican las tendencias ganadoras que pueden ser utilizadas para la formulación estratégica para que la radio online pueda plantear nuevas ventajas competitivas. 


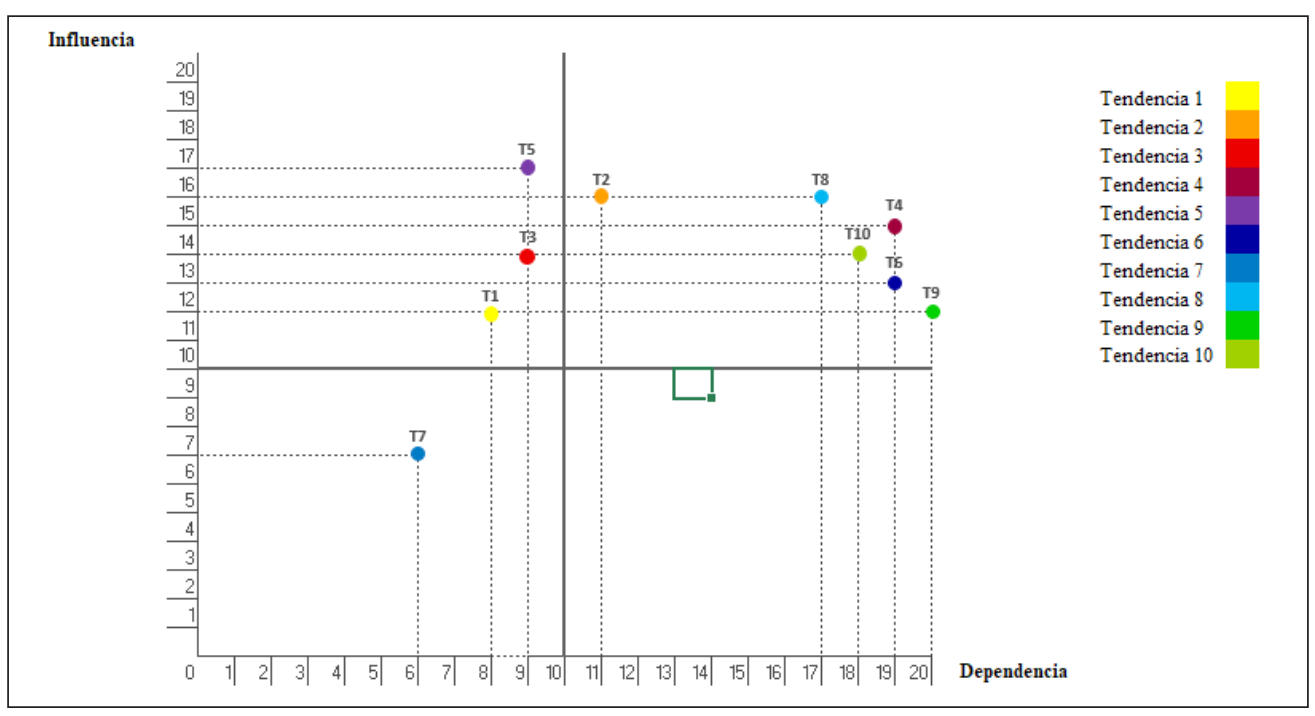

Figura 1. Clasificación de resultados tendencias.

Fuente: elaboración propia

\section{Tendencias ganadoras}

Se puede definir que en el cuadrante superior izquierdo las tendencias tienen una alta probabilidad en desarrollarse por ser totalmente influyentes y levemente dependientes, las tendencias ganadoras son la tendencia uno con relación al audio programático, la tendencia tres correspondiente a la mezcla de programaciones y finalmente la tendencia cinco sobre el branded content radiofónico. De acuerdo con los resultados las tres tendencias que se mencionaron anteriormente tienen un gran impacto el desarrollo de la radio online, el consumo de medios y la caracterización del oyente online.

\section{Audio programático}

La compra online surge como una nueva opción publicitaria de compra digital con el fin de ofrecer alternativas a los estrategas digitales que, sin importar la marca, producto o servicio puedan incrementar el tráfico, impactos, comercio electrónico y posicionamiento digital. Las oportunidades que ofrece el internet son infinitas, lo importante es que se cuente con los recursos correspondientes para lograr aprovecharlas, los procesos de comunicación han cambiado y las maneras en que una organización da a conocer los productos no son las mismas y los usuarios no los perciben de la misma manera, lo que implica que no es fácil diferenciarse y ofrecer una verdadera ventaja competitiva que permita diferenciarlos del resto de marcas de la categoría de mercado en la que participen.

De igual forma los consumidores se han transformado de consumidores en productores y consumidores de contenidos, los cuales no tienen ninguna restricción al momento de expresar sus opiniones y comentarios frente a la compra y consumo de los distintos 
productos, generando así un análisis crítico de los mismos y que los demás usuarios deberían conocer. Estos contenidos y comentarios pueden tener grandes repercusiones para las marcas lo cual produce algo de presión a las organizaciones de si en realidad debieran estar o no en línea. Las páginas web y la utilización de redes sociales son algunas de las estrategias que se utilizan para lograr una presencia de la marca a nivel online.

La compra programática permite que las marcas puedan lograr el máximo potencial digital, desde mejoras en la navegación de la página, incremento de tráfico u optimización de los motores de búsqueda. En ocasiones, las grandes inversiones digitales no se ven reflejadas en los resultados esperados o no son suficientes para ir más allá de la competencia. La publicidad online se caracteriza por ser más económica y más sencilla, para lograr el máximo potencial es importante definir el target, entender sus gustos, necesidades, motivaciones, cómo utilizan los medios digitales y que realizan en el tiempo en que están en línea; luego determinar qué se espera con las acciones digitales, entender el funcionamiento de la publicidad display y diferenciar entre el CPM o costo por mil impresiones, el CPC o costo por clics y el CPA o costo por acción. La primera permite que los anunciantes puedan definir el valor de cada mil anuncios publicados determinando las ubicaciones en donde estará la publicidad para impactar la mayor cantidad de usuarios, el segundo hace referencia al número de clics que pueden lograr los banners o formatos reach media para llevar a los usuarios al home page de la marca y, el tercero, es el pago que se realiza por la acción que un visitante realice en el site. Los audios programáticos consisten en la compra de anuncios en audio dentro de las aplicaciones Google Play Music, Spotify, SoundCloud, radios online o sitios de streaming de música o podcast, los cuales ofrecen una nueva alternativa para acceder a nuevos consumidores y fidelizar a los usuarios existentes logrando mayor efectividad de los anuncios, generando mayor valor para las marcas y los oyentes. Refleja una nueva oportunidad a través del análisis de datos del medio y del consumidor para la generación de campañas digitales.

\section{Mezcla de programaciones}

Desde el contexto de la sociedad de la información, el consumo de contenidos digitales ha crecido de manera exponencial, el uso de las TIC ha permitido la generación de entornos digitales centrados en la educación, procesos de formación, transmisión de información, crecimiento empresarial y entretenimiento por nombrar algunos. En el que el uso del internet a través de dispositivos como PC, tablets o móviles ha generado un crecimiento de los distintos contenidos digitales. Las organizaciones, las marcas y productos a través del marketing de contenidos han identificado una manera de no solo reconocer y producir contenidos, la estrategia surge para crear, publicar y distribuir contenidos con información de valor para los usuarios, el cual no tiene un fin comercial sino por el contrario pretende lograr una interacción directa con la marca.

Lograr la correcta generación de contenidos, que no solo atraiga a nuevos consumidores, trae una serie de ventajas para las marcas. Inicialmente, permite mejorar la percepción de los demás frente a la marca, tener la oportunidad de generar una relación directa con 
los consumidores de manera natural, fortalece el posicionamiento en los buscadores. Para lograr cada uno de estos elementos es importante conocer el consumo de medios digitales del target, en cuanto a los tiempos de consumo, motivaciones, actividades, plataformas, redes, comunidades, para lograr establecer qué tipo de contenidos se deben generar como: infografías, fotografías, post, frases, vídeos, paso a paso, preguntas, GIF animados, e-6ooks, newsletter, whitepaper; una vez identificados se debe planear la programación de contenidos, seguido del proceso de investigación con el fin de ofrecer información de valor y calidad para los consumidores y no pasar desapercibido como uno más. Para llevar a cabo cada uno de los contenidos se requiere de un presupuesto para definir el alcance que se desea lograr con los mismos y, por último, la variedad en la información presente en cada uno de los contenidos.

La radio online es uno de los medios que facilita el consumo de contenidos, los usuarios y oyentes digitales son cada vez más activos desde las redes sociales hasta los dispositivos móviles y logran generar una interacción directa con los comentaristas, programas, cadenas, hasta con otros oyentes. Las transformaciones tecnológicas han logrado que el medio esté presente constantemente en el día a día, logrando transmitir o compartir contenidos, dando oportunidad para la generación de contenidos interconectados que no solo mezclan las plataformas y medios de comunicación a través de los contenidos para lograr una identidad y diferenciarse del resto de los programas y cadenas radiales, todo ello facilita la personalización de contenidos y la generación de nuevas parrillas de programación a partir de los gustos y necesidades de los oyentes. El reto está en lograr la permanencia continua de los usuarios en la misma cadena radial.

\section{Branded content radiofónico}

Se entiende como la generación de contenidos de marca a partir del storytelling, dentro del inbound marketing permite que los usuarios encuentren a las marcas y no las marcas a los usuarios, aquí es donde el storytellingy y el arte de contar historias utilizan el relato y la narrativa para lograr una atmósfera que permita conectar emocionalmente con los usuarios y vivir una experiencia inolvidable. Parte de ello radica en qué tanto conocimiento se tiene del consumidor, qué les gustaría, qué información desean obtener, para luego determinar el tipo de contenido a generar y que historia desarrollar entorno a las marcas, diferenciándolo de la publicidad intrusiva que más que lograr una acción comercial se interpone en las necesidades digitales del usuario.

El branded content permite experiencias de valor, que más que informar los beneficios, cualidades y características de los productos pretende generar notoriedad y afinidad con los usuarios digitales para lograr engagement (relacionamiento), experiencias inolvidables de marca donde los usuarios tienen la oportunidad de expresar sus necesidades en relación con las marcas, personalizar cada una de sus experiencias, lograr la interacción a través de los múltiples contenidos que faciliten llegar a la audiencia, su desarrollo permite la creación de comunidades con personas afines a las marcas, con la generación de contenidos centrados en la marca y ser distribuidos desde la radio online permitirá a las más marcas, 
lograr mayor notoriedad y posicionamiento para lograr una identificación directa con los distintos productos y servicios. La creatividad debe ser un factor fundamental que permita el planteamiento de una campaña digital innovadora que logre una conexión emocional y directa con el consumidor dentro de la radio digital las plataformas audiovisuales son las más adecuadas para reflejar las emociones y experiencias que se viven en las cabinas de radio y los puntos de transmisión, con el soporte de concursos y eventos patrocinado por las marcas para lograr activaciones de marca, toma de sitios público o actividades BTL o Below the line sin dejar de lado uno de los componentes fundamentales del medio la programación musical, noticiosa o informativa que refleja el estilo del programa radial a nivel online.

Desde los programas de radio online se resalta 306Radio por ser pionera en el desarrollo de la radio online en el país, teniendo sus inicios en el año 2009 y logrando el reconocimiento como mejor emisora web por parte de Movistar y MinTIC en los premios Twitter Colombia 2011, se caracteriza por conocer y entender su audiencia, los millenials (nacidos entre 1982 y 2004); dentro de su programación se destacan los programas deportivos con información directa de los grupos de prensa ha equipos a nivel nacional, proponiendo una manera diferente de brindar información con contenidos innovadores.

\section{Conclusiones}

Las conclusiones en relación con el objetivo específico 1 -identificación de tendencias del medio a nivel digital con el fin de determinar su incidencia en la radio online-, son:

Como resultado de la evaluación de tendencias se permite identificar el crecimiento de la radio online y su adaptación a los cambios tecnológicos, políticos, económicos, sociales y culturales y cómo la formulación de estrategias de comunicación digital parte de las necesidades de las audiencias. Se valora la generación y el tipo de contenidos logrando ser más relevante que las plataformas en que se presentan, todo esto da a lugar al branded content como punto de partida en la formulación de estrategias de comunicación mediante la producción de contenidos de valor para lograr una conexión y vínculo significativo desde la radio online, esto significa que los contenidos deben ser más que relevantes para mejorar la experiencia con el medio y lograr establecer una credibilidad del mismo. Lo que presenta una oportunidad para las marcas y organizaciones para utilizar contenidos de valor significativos en la radio online como plataforma clave para extender los valores de la marca y los beneficios de los productos o servicios, todo ello centrado en las preferencias y comportamiento de los oyentes a partir de los estilos de vida.

Al generar el análisis de las treinta tendencias se identificaron las diez de mayor relevancia, que se caracterizan por tener una gran viabilidad de ejecución. Al explorar las categorías se halló que el mayor número de tendencias pertenecen al oyente online con un total de cinco, seguido del consumo de medios online con tres y finalmente en relación al medio se evidencian dos; lo cual indica que la formulación estratégica de la 
radio online se debe plantear desde las características, comportamientos, necesidades y motivaciones del oyente, como el centro de las acciones para la planeación de las parrillas de programación y contenidos.

La radio online se destaca como uno de los medios de mayor capacidad de adaptabilidad frente a los distintos retos que presenta el mercado, desde lo tecnológico, cultural, político, social y económico para lograr estar al aire, generar y transmitir información de valor a los oyentes sin importar el lugar, momento o plataforma. Lo que significa que la radio se ha transformado en un medio esencial para los usuarios ya que hace parte de su estilo de vida y facilita la generación de contenidos desde el oyente a través de comentarios, likes, recomendaciones, publicaciones entre otros.

Con base en el desarrollo de la matriz Micmac se determinó que las tendencias ganadoras que impactarán el medio a nivel digital son: los audios programáticos, la mezcla de programación y el branded content radiofónico como nuevas alternativas para el desarrollo de experiencias significativas. Al compararlas con la emisora digital 306Radio se puede concluir que están conscientes de la necesidad de conocer al oyente como el principal instrumento para la consolidación y posicionamiento de cada uno de los programas de la emisora.

El desarrollo de las nuevas tendencias permitirá que las cadenas de radio online puedan crear nuevas ventajas competitivas que permitan posicionar los programas desde la generación de contenidos de valor, logrando diversidad para impactar a la mayor cantidad de oyentes.

Las conclusiones en relación con el objetivo específico 2 -distinguir las transformaciones del medio desde lo tecnológico y digital-, son:

Unas de las grandes transformaciones que ha sufrido el medio, está en la manera en que los oyentes acceden a él, no solo se debe hablar sobre las frecuencias u ondas de radio, sino de nuevas herramientas y plataformas de difusión como streaming y podcast, lo cual amplía el rango de impacto a nuevas audiencias y nuevos contenidos para lograr un mayor interés hacia el medio a nivel digital.

La diversificación de contenidos ha generado que una variedad en relación con el canal de comunicación a utilizar desde las redes sociales, páginas web, blogs y e-mail marketing, lo cual permite que el medio pueda reinventarse desde los contenidos para llegar de una manera diferente pero efectiva a la audiencia, al punto que se puede lograr la personalización de estos para lograr un relacionamiento o engagement con las emisoras.

A su vez, la convergencia de los medios de comunicación, entre ellos la radio, presenta un reto constante para las emisoras digitales, desde el lenguaje y comportamientos en donde las audiencias tradicionales se adaptan a las trasformaciones digitales generando un nuevo oyente, que propone nuevos usos y hábitos de consumo. 
Las conclusiones en relación con el objetivo específico 3 -reconocer las estrategias digitales a utilizar para orientar las acciones del medio online-, son:

La formulación estratégica de la radio a nivel digital tiene incidencia desde el portal web en donde se aloje la emisora, la parrilla de programación, la generación de contenidos, las redes sociales y, finalmente, la manera en que llegan a la audiencia.

La facilidad de navegación y acceso a la información es un elemento determinante para el oyente de la emisora digital, la estrategia debe enfocar la emisora como el centro de la información y contenidos que desee el usuario para garantizar el mayor tiempo de estadía e interacción con la marca, por ello cada vez más las emisoras digitales producen contenidos complementarios a las parillas de programación, se transforman en voceros y representantes de los oyentes en distintos eventos musicales para vincular al oyente directamente con la emisora digital, invitando a los usuarios a compartir y comentar en las distintas redes sociales, cada una de estas acciones y estrategias marcan un nuevo consumo del medio.

Finalmente, una de las grandes estrategias que se identifica es la personalización de los playlist desde las temáticas planteadas por las emisoras digitales, lo que conlleva una relación directa de cada uno de los contenidos generados desde el medio, en donde no solo está el programa radial, sino la selección musical relacionada con la temática, revisión audiovisual de bandas, videos y letras, permiten nuevas maneras de interacción con la audiencia lo cual incrementa su participación en redes sociales.

\section{Referencias}

Acebes, B. y Borrego, R. (2018). Estudio de audio online 2018 (III). Recuperado de http://www.dosdoce. com/wp-content/uploads/2018/05/Estudio-del-audio-online-iabspain-2018.pdf

Arnau, J. (1996). Métodos y técnicas avanzadas de análisis de datos en ciencias del comportamiento. Barcelona: Universitat.

Barrios, A. (2013). La radio en la era de la sociedad digital. adComunica. Revista de Estrategias, Tendencias e Innovación en Comunicación, (5), 37-54.

Bitar, D. (2018). Radio: cinco tendencias que marcarán el futuro. Recuperado de http://www.revistapym. com.co/radio-cinco-tendencias-marcaran-futuro

Corporación Colombia Digital. (Septiembre 26 de 2017). Colombia Digital. Recuperado de https:// colombiadigital.net/actualidad/bytes/item/9862-5-tendencias-que-cambiaran-la-radio-como-laconocemos.html

Costa, M. D., Moreno, E. y Amoedo, A. (2012). La radio generalista en la red: un nuevo modelo para la radio tradicional. Anagramas, 10(20), $165-180$.

Crettaz, J. (2103). Radios online: un fenómeno nuevo que se hace escuchar. Recuperado de https://www. lanacion.com.ar/1546747-radios-online-un-fenomeno-nuevo-que-se-hace-escuchar

García, T. y García, A. (2001). Medios de comunicación, sociedad y educación. Castilla:Universidad de Castilla. 
González, M. (2010). La ciberradio: nueva alternativa de futuro para la radio. Juventud y nuevos medios de comunicación, (88), 51-62.

Godet, M. (2007). Prospectiva estratégica: problemas y métodos. Recuperado de http://www. laprospective.fr/dyn/francais/memoire/Cajadeherramientas2007.pdf

Herreros, M. C. (2009). Comunicación interactiva en los cibermedios. Comunicar Revista Científica de Educomunicación, 17(33), 15-24.

Levy, P. (2007). Cibercultura. La cultura en la sociedad digital. Educatio Siglo XXI, (26), 295-298. Recuperado de http://revistas.um.es/educatio/article/viewFile/46731/44761

Martínez, M. y Prata, N. (2017). La radio en busca de su audiencia: hacia una escucha diversificada y multiplataforma. Intercom-RBCC, 40(3), 109-128. doi: 10.1590/1809-5844201737

Ministerio de Tecnologías de la Información y las Comunicaciones. MINTIC. (2018). Boletín trimestral de las TIC. Recuperado de https://colombiatic.mintic.gov.co/679/articles-75854_archivo_pdf.pdf

Orihuela, J. (2015). Los medios después de internet. Barcelona: Universitat Oberta de Catalunya.

Romero, H. (2011). La digitalización de la radio deportiva. Fonseca, Journal of Communication, 3, 132-152.

Scolari, C., Winocur, R., Pereira, S. y Barrechene, C. (2018). Alfabetismo transmedia. Recuperado de http://www.comunicacionysociedad.cucsh.udg.mx/index.php/comsoc/article/view/7227/5998

Socelec, S. (2017). La conectividad y el internet de las cosas. Energética XXI, 41.

Tigre, R. (22 de marzo de 2018). Las 10 tendencias del audio digital para 2018. Recuperado de http:// blog.audio.ad/es/2017/12/las-10-tendencias-del-audio-digital-para-2018/ 